\title{
Physiology, phylogeny, and LUCA
}

\author{
William F. Martin*,1,2 ${ }^{*}$ Madeline C. Weiss ${ }^{1}$, Sinje Neukirchen ${ }^{3}$, Shijulal Nelson-Sathi ${ }^{4}$, Filipa L. Sousa ${ }^{3}$ \\ ${ }^{1}$ Institute for Molecular Evolution, Heinrich-Heine Universität Düsseldorf, Universitätstrasse 1, 40225 Düsseldorf, Germany. \\ ${ }^{2}$ Instituto de Tecnologia Química e Biológica, Universidade Nova de Lisboa, 2780-157 Oeiras, Portugal. \\ ${ }^{3}$ Department of Ecogenomics and Systems Biology, University of Vienna, Althanstrasse 14, 1090 Vienna, Austria. \\ ${ }^{4}$ Computational Biology \& Bioinformatics Group, Rajiv Gandhi Centre for Biotechnology (RGCB), Trivandrum, Kerala 695014, India. \\ * Corresponding Author: \\ William F. Martin, Molekulare Evolution, Heinrich-Heine-Universität Düsseldorf, Building: 26.13, Floor/Room: 01.34, \\ Universitätsstraße 1; 40225 Duesseldorf, Germany; Tel: +49 21181 13011; Fax: +49 21181 13554; E-mail: bill@hhu.de
}

\begin{abstract}
Genomes record their own history. But if we want to look all the way back to life's beginnings some 4 billion years ago, the record of microbial evolution that is preserved in prokaryotic genomes is not easy to read. Microbiology has a lot in common with geology in that regard. Geologists know that plate tectonics and erosion have erased much of the geological record, with ancient rocks being truly rare. The same is true of microbes. Lateral gene transfer (LGT) and sequence divergence have erased much of the evolutionary record that was once written in genomes, and it is not obvious which genes among sequenced genomes are genuinely ancient. Which genes trace to the last universal ancestor, LUCA? The classical approach has been to look for genes that are universally distributed. Another approach is to make all trees for all genes, and sift out the trees where signals have been overwritten by LGT. What is left ought to be ancient. If we do that, what do we find?
\end{abstract}

doi: $10.15698 /$ mic2016.12.545

Received originally: 21.09.2016;

in revised form: 27.09.2016,

Accepted 28.09.2016.

Published 25.11.2016.

Keywords: early evolution, autotrophy, geochemistry, acetogens, methanogens.
Early evolution and the nature of the very first kinds of life are interesting topics. They concern the phase of Earth history where our most distant ancestors emerged from the elements on an otherwise lifeless planet. The questions of how the initial evolutionary transition - from inanimate to animate matter - might have happened and what the first kinds of life were like in terms of habitat and lifestyle are just plain interesting. People generally want to know about how things were in the past, including the most distant past. It is apparently part of human nature to wonder where we came from.

An important concept in very early evolution is the last universal common ancestor, LUCA for short, because it represents the organism, cell, thing, or chemical reaction, depending on one's concept of LUCA, from which all life forms we know are descended. Thoughts about the nature of LUCA abound in the literature and are immensely diverse; the search term 'last universal common ancestor' alone returns 188 articles since 1997 in standard literature databases. Diversity of thoughts on LUCA is partly due to the circumstance that when we, as scientists, conceptually delve as deep as LUCA in evolutionary history, we are not far removed from the topic of life's origin. Thoughts on the origin of life are even more diverse than on LUCA, with over 2200 articles in literature databases appearing with 'origin of life' as the query. How can one learn more about the biology of LUCA, the starting point of early evolution?

If we look around, there are presently only two ways to empirically approach early evolution: geology and genomes. A prominent geologist, Andy Knoll, likes to say "Earth records its own history" [1], which is spot-on. Geology can indeed tell us when life arose. The oldest sedimentary rocks, which are ca. 3.8 billion years of age, harbour traces for life in the form of light carbon isotopes, evidence for biological $\mathrm{CO}_{2}$ fixation at that time $[2,3]$. But the presence of $\mathrm{CO}_{2}$ fixation, possibly even as far back as $4.1 \mathrm{Ga}$ [4] does not tell us everything that we might want to know about early life. Indeed, plate tectonics and erosion have erased much of the Earth's recorded history, with truly ancient rocks being rare and their evidence for early life often being difficult to interpret. Nonetheless, the geochemical record does harbor evidence for physiological processes.

A problem arises, though, in that physiological processes among prokaryotes are not generally restricted to any particular phylogenetic group. A glaring exception to that rule are the cyanobacteria, who also infringe upon the rule that Earth records its own history, because since cyanobacteria have been around, they have been editing a lot of Earth's recorded text with their waste product, oxygen [5]. Outside of the cyanobacteria, phylogeny and physiology are decoupled by the reality of lateral gene transfer (LGT) 
among prokaryotes: sulfate reduction [6], anoxygenic photosynthesis [5], fermentations [7], and respirations [8] are distributed among many different prokaryotic lineages, but because of LGT, not because of differential loss: LUCA could not do everything, it can hardly have possessed a genome of Eden. One might interject that methanogenesis is restricted to a particular phylogenetic group, the methanogens, but new phylogenetic depictions of the 'tree of life' have methanogens basal among the archaea, with loss of methanogenesis in many independent groups $[9,10]$, those losses corresponding to gene acquisitions from bacteria in some cases [11], thereby decoupling phylogeny from physiology in the methanogens, too, which no longer appear as a monophyletic group.

Curiously, genomes also record their own history. But lateral gene transfer (much like plate tectonics) and sequence divergence (much like erosion) have erased much of the evolutionary signal that the very first genomes on our planet contained. Nonetheless we can be sure that there was a time and a place and an environment where those very first genomes did exist. How can one harness genomes to find out more about what the first life forms were like, and how to get a better picture of LUCA?

In the modern era (since the discovery of archaea), the ribosomal RNA tree of life, or the three domain tree [12], has been the main starting point for inferences about the nature of LUCA. But as progress has accrued with genomes, three issues have come to the fore that bear on inferences of LUCA's gene set: i) the effects of lateral gene transfer on our picture of LUCA, ii) the question of whether the three domain tree is correct, and iii) the issue of how universally distributed genes need to be in order to trace to LUCA.

The LGT issue is fairly straightforward. One avenue of investigation into LUCA has been to see which, what kind of and how many genes are common to archaea, bacteria and eukaryotes (all three domains). All things being equal, and barring LGT, such genes would trace to LUCA. So by simply looking for gene presence, Ouzounis et al. [13] could attribute about 1000 genes to LUCA, if LUCA was taken as the common ancestor of prokaryotes, or up to 1400 genes, if eukaryotes were included and if one allowed for widespread gene loss and excluded LGT. But like earlier investigations [14] and later investigations [15], Ouzounins et al. [13] attributed all absences of genes among lineages descended from LUCA to differential loss. If genes were distributed across domains by LGT, rather than differential loss, then presence of a gene in all three domains (or in both prokaryotic domains) would not reflect presence in LUCA, it would just reflect transdomain LGT. If not identified and removed, LGT generates overestimates of LUCA's gene content. Kannan et al. [16] very clearly spelled out the problem that transdomain LGT introduces into the study of LUCA's genes, and they also explained why it is not trivial to circumvent the LGT problem. The real problem with transdomain LGT is not that it has been known for many years to be an issue in early evolution [17], rather the real issue is its prevalence in nature today and in the past. Phylogenetic studies spanning all genes from many hundreds of genomes uncover thousands of cases of transdomain LGT, mainly from bacteria to archaea $[11,18]$. If such LGT cases are identified and filtered out, maybe a picture of LUCA will come into focus.

The influence of the three domain tree on the issue of LUCA is somewhat more complicated. Many investigators on the issue of LUCA have adhered strictly to the three domain tree, meaning that if one wants to address LUCA, one must first place a root somewhere on the three domain tree. Investigations of anciently duplicated genes $[19,20]$ led to placement of the root on the bacterial branch [12]. But even among proponents of the three domain tree, the bacterial root was not universally accepted. For example, there have been strong proponents of the view that, the three domain tree is correct, but its root should be on the eukaryotic branch, coupled with the view that LUCA was more similar to eukaryotes than it was to prokaroytes [21-24] - a line of inference that has led its proponents to argue that the term 'prokaryote' be banned from the literature altogether. Di Guilio [25] also argues that we should ban the use of the term prokaryotes, albeit on grounds that do not hinge upon arguments that the first cells were eukaryote-like. Such discussions result in suggestions for terms like acaryotes, akaryotes, arkarya, and syncaryote [26] to replace the very useful concepts of prokaryotes and eukaryotes, terms which the more physiologically minded among us [27] are (wisely, we think) unwilling to surrender.

While debates about LUCA and higher order microbial nomenclature have been brewing, something else far more threatening for the three domain tree has been gnawing on its trunk: the three domain tree apparently has the domain relationships wrong. Recently, a small revolution in deep phylogenetic views has occurred, with newer methods of phylogenetic inference and investigations based on broader sampling of archaeal lineages having brought forth a new view of domain relationships, in which the archaeal component of eukaryotes branches within the archaea, not as a sister to them [9, 28-32]. Jim Lake will be quick to point out that some people had been saying that for 30 years [33]. Defenders of the three domain tree counter that there is no need to worry, the three domain tree will persist [34]. But people keep on finding the new tree of domain relationships, which is currently being called the two domain tree [29]. Lake [33] (1988) called it the eocyte tree but the name did not stick well. In the two domain tree - which incidentally fits very well with what some of us have been saying about eukaryote origin for a long time [35] - genes that trace to LUCA need not be present in eukaryotes at all. That is because in the two domain tree, eukaryote genomes arose from a very small sample of prokaryotic gene diversity, in the simplest case from the symbiotic association of two prokaryotic genomes in the form of an archaeal host with a bacterial symbiont, the ancestor of mitochondria and hydrogenosomes [36, 37].

Related to the issue of the three domain tree is the issue of how universal gene distributions need to be to trace a gene to LUCA. Regardless of where the root is, one can still look for genes that trace to LUCA by virtue of the density of their distribution. If one is strict, requiring that 
genes be universally distributed across genomes, about 3036 genes trace to LUCA [38-40]; if one allows for a bit of loss, about 100 genes trace to LUCA [41]; if one allows for a bit more loss, then about 500-600 genes trace to LUCA [42]; and if we allow for a lot of loss, then we are redirected to the issue above, namely that presence/absence patterns might be due to transdomain LGT rather than to differential loss, such that simple presence of a gene in bacteria and one archaeon or vice versa [15] is not solid ground for saying that said gene was present in LUCA.

In addition, if LUCA's gene set is defined in such a way that has to include genes that are present in eukaryotes (by the criterium of being present in three domains), then we quickly end up with an inference of LUCA that had a glycolytic pathway [42] and that used oxygen as a terminal acceptor [23], because that is how most eukaryotes obtain their energy [43]. But we know from physiology that the first free-living cells cannot have been chemoorganotrophs (satisfying their energy needs by the oxidation or disproportionation of reduced carbon compounds) because organics from space are nonfermentable substrates [44]. We also know from physiology that the producers of oxygen, cyanobacteria, represent a bioenergetically very advanced stage in physiological evolution $[45,46]$, and thus cannot have preceded LUCA to generate oxygen for it to breathe. We also know from physiology that the mitochondria of many eukaryotes do not require oxygen for ATP synthesis [36].

Aware of the foregoing, we recently undertook a phylogenetic investigation based upon the two domain tree in search of insights into LUCA that might illuminate its microbial lifestyle [47]. Rather than looking for genes that are universally distributed (or nearly universally distributed), we looked for genes that trace to LUCA by virtue of being ancient. As our criterion for ancient, we looked for genes that are present in bacteria and archaea, but not because of LGT. This approach embraces the two domain tree, in which eukaryotes have nothing to do with life's origin, thereby excluding eukaryotes from the analysis. But how to exclude LGT? We looked for genes that fulfill two very simple criteria: i) the gene is present in two members each of two major groups of archaea and bacteria and ii) the domains are monophyletic. Genes that fulfill those criteria are unlikely to have a distribution that results from LGT.

In order to identify such genes, there is presently no obvious alternative to making all trees for all genes in all sequenced genomes and separating the wheat (the trees that show domain monophyly in the two domain tree) from the chaff (the trees that show archaea and bacteria interleaving). We have been making trees for large numbers of genes for some time [11,18, 48-50]. Trees for all genes are important because it has become evident that in prokaryotes, each gene has its own independent evolutionary history and that "trees of life", whether based on rRNA or the currently popular collection of ribosomal proteins $[29,30,38]$ are not good proxies for what genes will be present in the rest of the genome and how those genes will be related to homologues from other genomes, because LGT is very prevalent among prokaryotes.
When we were done sorting the trees, what we found in our analysis were 355 genes that depict LUCA as an anaerobic autotroph that lived in a hot, gas-rich, metal-rich environment [47]. Its inferred energy metabolism was dependent upon $\mathrm{H}_{2}$ and $\mathrm{CO}_{2}$, it could fix $\mathrm{N}_{2}$, it had a heavy dependence upon transition metals, its metabolism revealed an extremely prominent role for methyl groups, one electron transfers, radical reactions, and redox chemistry. Its carbon metabolism was based on the acetyl-CoA pathway, the oldest of the six known $\mathrm{CO}_{2}$ fixation pathways. It was capable of substrate level phosphorylation using the acetyl-CoA pathway and it could harness chemiosmotic potential. It had modified bases, mostly involving methylations, suggesting that not only LUCA, but also the genetic code arose in an environment where reactive methyl groups were abundant. Previous studies had uncovered little information about LUCA's physiology and habitat. That is probably because earlier studies had focused on genes that are universally distributed (or nearly so). We also found that the trees of genes that trace to LUCA implicate clostridia (which harbour many acetogens) and methanogens as the earliest-branching forms of bacteria and archaea respectively. That fits with the functions of the genes we found, because acetogens and methanogens have carbon and energy metabolism that depends upon $\mathrm{H}_{2}$ and $\mathrm{CO}_{2}$, they can fix $\mathrm{N}_{2}$, they have a heavy dependence upon transition metals, and their core physiology reveals an extremely prominent role for methyl groups, one electron transfers, radical reactions, and redox chemistry.

The results that we obtained fit very well with the idea that life arose in submarine hydrothermal vents and that the first cells were autotrophs that satisfy both their carbon and their energy needs from the reduction of $\mathrm{CO}_{2}$ with electrons from $\mathrm{H}_{2}$ [51-53]. Notably, $\mathrm{H}_{2}$ is still continuously generated in modern hydrothermal vents today by the process of serpentinization [54], a spontaneous and exergonic geochemical reaction in which $\mathrm{Fe}^{2+}$ in oceanic crust reduces $\mathrm{H}_{2} \mathrm{O}$ to generate $\mathrm{H}_{2}$, which can reach many concentrations in vent effluent of many millimols per liter [55]. We found no evidence for a role of photosynthesis in LUCA's physiology, in particular there was no evidence for ZnS-based photosynthesis in LUCA (a physiology that is unknown among modern life forms anyway), in contrast to the predictions of some other recent theories [56]. Rather we found evidence linking LUCA to known forms of microbial physiology - acetogenesis and methanogenesis without cytochromes [57] - that are manifest among the strictest anaerobes [58,59], with evidence for a role of sulfur metabolism [60], and with a very important role for $\mathrm{Fe}, \mathrm{Ni}, \mathrm{Mo}$, and $\mathrm{Co}$, transition metals that play a central role in the metabolism of anaerobic autotrophs today.

Our recent findings depart from phylogeny-based views of LUCA germane to the three domain tree and uncover connections between modern microbial physiology and geochemical environments on the early Earth. Some will surely complain that 355 genes is not enough and that essential functions like lipid synthesis, amino acid and nucleotide biosyntheses are very poorly represented in LUCA's gene set. How can anything live without that? As we 
wrote, lack of such essential functions among LUCA's gene set could indicate i) that the missing genes unspectacularly underwent transdomain lateral gene transfer (LGT) postLUCA and hence were filtered out by our method, ii) that some missing chemical components were provided by spontaneous abiotic syntheses during early Earth history, or iii) a combination thereof. Transdomain LGT is both normal and natural, and all theories for the origin of cells, without exception, require abiotic syntheses, hence we do not see any fundamental problems in that regard. There was a time on the early Earth when there was no life and there was a time when there was life. If we filter out the effects of 4 billion years of LGT - which is, in essence, what we did - a picture of LUCA emerges that represents something that was half-alive, an intermediate in the transition from rocks and water on a young, barren planet to something that could scratch a living out of gasses and mineral salts. For some reason, that sounds quite reasonable to us, others will surely disagree.

It is very interesting that acetogens and methanogens inhabit the crust today $[10,61]$. Geochemists say that the convective currents of water that permeate the Earth's crust to drive serpentinization have been going on since there was water on Earth [62]. Let us presume, just for a moment, that the first bacteria and archaea were acetogens and methanogens respectively. On an uninhabited planet, they have no competitors, and life multiplies quickly given ample growth substrates. The founders of their respective domains would have bubbled off into the ocean bottom waters to be spread around by currents and eventually to be introduced back into hydrothermal systems in the crust, where they would have found the diet that they were raised on. It is possible that some anaerobic autotrophs that live from the reduction of $\mathrm{CO}_{2}$ with $\mathrm{H}_{2}$ still inhabit the same niche in which life arose, albeit not the same rocks because during Earth history oceanic crust is constantly recycled into the mantle via subduction. In that sense, acetogens and methanogens really might provide a glimpse into the biology of the very first microbes on Earth, as some microbiologists familiar with the physiology of

\section{REFERENCES}

1. Gaidos E, and Knoll AH (2012). Our evolving planet: From dark ages to evolutionary renaissance. In: Impey C, Lunine J, Funes J, editors. Frontiers of Astrobiology. Cambridge University Press, Cambridge; pp 132-153.

2. Mojzsis SJ, Arrhenius G, McKeegan KD, Harrison TM, Nutman AP and Friend CR (1996). Evidence for life on Earth before 3,800 million years ago. Nature 384: 55-59.

3. Ueno $Y$, Yurimoto $H$, Yoshioka H, Komiya $T$, and Maruyama S (2002). Ion microprobe analysis of graphite from ca. 3.8 Ga metasediments, Isua crustal belt, West Greenland: Relationship between metamorphism and carbon isotopic composition. Geochimia Et Cosmochimia Acta 66(7):1257-1268.

4. Bell EA, Boehnke P, Harrison TM, and Mao WL (2015). Potentially biogenic carbon preserved in a 4.1 billion-year-old zircon. Proc Nat Acad Sci U S A 112(47):14518-14521. these organisms have been saying for some time $[45,60$, 63].

Over four decades ago, biochemists thought that FeS clusters are ancient [64] and that acetogens and methanogens are ancient [45], based on good intuition, common sense, and some straightforward principles of physiology. With the discovery of archaea, the three domain tree led to avenues of thought about early evolution that were guided by phylogeny rather than physiology. LGT conflates phylogeny. But LGT does not conflate physiology, it just decouples it from phylogeny. When we filter out the LGT from all of the gene trees that we can make from genomes, we end up with a picture of LUCA that looks very much like what experts familiar with the physiology of anaerobes had in mind in the late 1960's [45], and still have in mind today $[65,66]$. If we return to the geochemical record, the first evidence for life we see is evidence for autotrophs $[3,4]$, which is also what genomes recently uncovered about LUCA [47]. Thus, on the issue of autotrophs being ancient, geology and physiology converge. The version of LUCA that is obtained by taking all the data and simply removing the obvious LGT interfaces well with Earth history, with microbial physiology, and even with the new two domain tree. It also bears out the predictions of some specific formulations the theory that life arose at submarine hydrothermal vents.

\section{CONFLICT OF INTEREST}

The authors declare no conflict of interest.

\section{COPYRIGHT}

(C) 2016 Martin et al. This is an open-access article released under the terms of the Creative Commons Attribution (CC BY) license, which allows the unrestricted use, distribution, and reproduction in any medium, provided the original author and source are acknowledged.

Please cite this article as: William F. Martin, Madeline C. Weiss, Sinje Neukirchen, Shijulal Nelson-Sathi, Filipa L. Sousa (2016). Physiology, phylogeny, and LUCA. Microbial Cell 3(12): 582-587. doi: 10.15698/mic2016.12.545

5. Fischer WW, Hemp J, and Johnson JE (2016). Evolution of oxygenic photosynthesis, Ann Rev Earth Planet Sci 44:647-683.

6. Rabus R, Venceslau SS, Wöhlbrand L, Voordouw G, Wall JD, and Pereira IAC (2015). A post-genomic view of the ecophysiology, catabolism and biotechnological relevance of sulphate-reducing prokaryotes. Adv Microb Physiol 66:55-321.

7. Barker HA (1961). Fermentation of nitrogenous compounds. In: Gunsalus IC and Stanier RY, editors. The Bacteria. A Treatise on Structure and Function. Academic Press, New York pp. 151-207.

8. Marreiros BC, Calisto F, Castro PJ, Duarte AM, Sena FV, Silva AF, Sousa FM, Teixeira M, Refojo PN, and Pereira MM (2016). Exploring membrane respiratory chains. Biochim Biophys Acta 1857(8):10391067. 
9. Raymann K, Brochier-Armanet C, and Gribaldo S (2015). The twodomain tree of life is linked to a new root for the Archaea. Proc Nat Acad Sci U S A 112:6670-6675.

10. Evans PN, Parks DH, Chadwick GL, Robbins SJ, Orphan VJ, Golding $\mathrm{SD}$, and Tyson GW (2015). Methane metabolism in the archaeal phylum Bathyarchaeota revealed by genome-centric metagenomics. Science 350:434-438.

11. Nelson-Sathi S, Sousa FL, Roettger $M$, Lozada-Chávez $N$, Thiergart $T$, Janssen A, Bryant D, Landan G, Schönheit P, Siebers B, Mclnerney JO, and Martin WF (2015). Origins of major archaeal clades correspond to gene acquisitions from bacteria. Nature 517:77-80.

12. Woese CR, Kandler O, and Wheelis ML (1990). Towards a natural system of organisms: proposal for the domains Archaea, Bacteria, and Eucarya. Proc Natl Acad Sci U S A 87: 4576-4579.

13. Ouzounis CA, Kunin V, Darzentas N, and Goldovsky L (2006). A minimal estimate for the gene content of the last universal common ancestor-exobiology from a terrestrial perspective. Res Microbiol 157:57-68.

14. Castresana J (2001). Comparative genomics and bioenergetics. Biochim Biophys Act - Bioenerg 1506:147-162.

15. Nitschke W, and Russell MJ (2013). Beating the acetyl coenzyme Apathway to the origin of life. Phil Trans Roy Soc Lond B. 368:20120258.

16. Kannan L, Li H, Rubinstein B, and Mushegian A (2013). Models of gene gain and gene loss for probabilistic reconstruction of gene content in the last universal common ancestor of life. Biol Direct 8:32.

17. Martin W, and Cerff R (1986). Prokaryotic features of a nucleus encoded enzyme: cDNA sequences for chloroplast and cytosolyic glyceraldehyde-3-phosphate dehydrogenases from mustard (Sinapis $a / b a)$. Eur J Biochem 159:323-331.

18. Nelson-Sathi S, Dagan T, Landan G, Janssen A, Steel M, Mclnerney JO, Deppenmeier U, and Martin WF (2012). Acquisition of 1,000 eubacterial genes physiologically transformed a methanogen at the origin of Haloarchaea. Proc Natl Acad Sci U S A 109:20537-20542.

19. Iwabe N, Kuma K, Hasegawa M, Osawa S, and Miyata T (1989). Evolutionary relationship of archaebacteria, eubacteria, and eukaryotes inferred from phylogenetic trees of duplicated genes. Proc Natl Acad Sci U S A 86:9355-9359.

20. Gogarten JP, Kibak H, Dittrich P, Taiz L, Bowman EJ, Bowman BJ, Manolson MF, Poole RJ, Date T, Oshima T, Konishi J, Denda K, and Yoshida M (1989). Evolution of the vacuolar $\mathrm{H}^{+}$-ATPase: Implications for the origin of eukaryotes. Proc Natl Acad Sci U S A, 86:6661-6665.

21. Forterre $P$ (1995). Thermoreduction, a hypothesis for the origin of prokaryotes. C R Acad Sci III 318:415-422.

22. Poole A, Jeffares D, and Penny D (1999). Early evolution: prokaryotes, the new kids on the block. BioEssays 21: 880-889.

23. Glansdorff N, Xu Y, and Labedan B (2008). The Last Universal Common Ancestor: emergence, constitution and genetic legacy of an elusive forerunner. Biol Direct 3:29.

24. Harish A, Tunlid A, and Kurland CG (2013). Rooted phylogeny of the three superkingdoms. Biochimie. 95:1593-1604.

25. Di Giulio M (2015). The non-biological meaning of the term "Prokaryote" and its implications. J Mol Evol 80:98-101.

26. Forterre $P$ (2015). The universal tree of life: an update. Front Microbiol 6:717.

27. Whitman EB (2009). The modern concept of the procaryote. J Bact 191:2000-2005.
28. Cox CJ, Foster PG, Hirt RP, Harris SR and Embley TM (2008). The archaebacterial origin of eukaryotes. Proc Nat Acad Sci U S A 105:20356-20361.

29. Williams TA, Foster PG, Cox CJ, and Embley TM (2013). An archaeal origin of eukaryotes supports only two primary domains of life. Nature 504:231-236.

30. Spang A, Saw JH, Jørgensen SL, Zaremba-Niedzwiedzka K, Martijn J, Lind AE, van Eijk R, Schleper C, Guy L, and Ettema TJ (2015). Complex archaea that bridge the gap between prokaryotes and eukaryotes. Nature 521:173-179.

31. Mclnerney J, Pisani D, and O'Connell MJ (2015). The ring of life hypothesis for eukaryote origins is supported by multiple kinds of data. Phil Trans R Soc B 370:20140323.

32. Hug LA, Baker BJ, Anantharaman K, Brown CT, Probst AJ, Castelle CJ, Butterfield CN, Hernsdorf AW, Amano Y, Ise K, Suzuki Y, Dudek N, Relman DA, Finstad KM, Amundson R, Thomas BC, and Banfield JF (2016). A new view of the tree of life. Nature Microbiol 1:16048.

33. Lake JA (1988). Origin of the eukaryotic nucleus determined by rate-invariant analysis of rRNA sequences. Nature 331:184--186.

34. Forterre $P$ (2013). The common ancestor of archaea and eukarya was not an archaeon. Archaea. 2013: 372396.

35. Martin W, and Müller M (1998). The hydrogen hypothesis for the first eukaryote. Nature 392:37-41.

36. Müller M, Mentel M, van Hellemond JJ, Henze K, Woehle C, Gould SB, Yu R-Y, van der Giezen M, Tielens AGM, and Martin WF (2012). Biochemistry and evolution of anaerobic energy metabolism in eukaryotes. Microbiol Mol Biol Rev 76:444-495.

37. Sousa FL, Neukirchen S, Allen JF, Lane N, and Martin WF (2016). Lokiarchaeon is hydrogen dependent. Nature Microbiol. 1:16034.

38. Hansmann S, and Martin W (2000). Phylogeny of 33 ribosomal and six other proteins encoded in an ancient gene cluster that is conserved across prokaryotic genomes: influence of excluding poorly alignable sites from analysis. Int J Syst Evol Microbiol 50(4):1655-1663.

39. Charlebois RL, and Doolittle WF (2004). Computing prokaryotic gene ubiquity: Rescuing the core from extinction. Genome Res 14: 2469-2477.

40. Ciccarelli FD, Doerks T, Mering von C, Creevey CJ, Snel B, and Bork $P$ (2006). Toward automatic reconstruction of a highly resolved Tree of Life. Science 311, 1283-1287.

41. Puigbò $P$, Wolf $\mathrm{YI}$, and Koonin EV (2009). Search for a 'Tree of Life' in the thicket of the phylogenetic forest. J Biol 8:59.

42. Koonin EV (2003). Comparative genomics, minimal gene-sets and the last universal common ancestor. Nat Rev Microbiol 1: 127-136.

43. Lane N, and Martin W (2010). The energetics of genome complexity. Nature 467:929-934.

44. Schönheit P, Buckel W, and Martin WF (2016). On the origin of heterotrophy. Trends Microbiol 24:12-25.

45. Decker K, Jungerman K, and Thauer RK (1970). Energy production in anaerobic organisms. Angew Chem Int Ed. 9:138-158.

46. Martin WF, and Sousa FL (2016). Early microbial evolution: the age of anaerobes. Cold Spring Harbor Persp Biol 8:a018127.

47. Weiss MC, Sousa FL, Mrnjavac N, Neukirchen S, Roettger M, Nelson-Sathi S, and Martin WF (2016). The physiology and habitat of the last universal common ancestor. Nature Microbiol 1(9):16116.

48. Martin W, Stoebe B, Goremykin V, Hansmann S, Hasegawa M, and Kowallik KV (1998). Gene transfer to the nucleus and the evolution of chloroplasts. Nature 393:162-165. 
49. Martin W, Rujan T, Richly E, Hansen A, Cornelsen S, Lins T, Leister D, Stoebe B, Hasegawa M, and Penny D (2002). Evolutionary analysis of Arabidopsis, cyanobacterial, and chloroplast genomes reveals plastid phylogeny and thousands of cyanobacterial genes in the nucleus. Proc Natl Acad Sci U S A 99:12246-12251.

50. Ku C, Nelson-Sathi S, Roettger M, Sousa FL, Lockhart PJ, Bryant D, Hazkani-Covo E, Mclnerney JO, Landan G, and Martin WF (2015). Endosymbiotic origin and differential loss of eukaryotic genes. Nature 524:427-432.

51. Russell MJ, and Martin W (2004). The rocky roots of the acetyl-CoA pathway. Trends Biochem Sci 29:358-363.

52. Martin W, and Russell MJ (2007). On the origin of biochemistry at an alkaline hydrothermal vent. Phil Trans Roy Soc Lond B 367:18871925.

53. Lane N, and Martin WF (2012). The origin of membrane bioenergetics. Cell 151:1406-1416.

54. Russell MJ, Hall AJ, and Martin W (2010). Serpentinization as a source of energy at the origin of life. Geobiol 8:355-371.

55. Schrenk MO, Brazelton WJ, and Lang SQ (2013). Serpentinization, carbon, and deep life. Rev Mineral Geochem 75:575-606.

56. Mulkidjanian AY, and Galperin MY (2009) On the origin of life in the zinc world. 2. Validation of the hypothesis on the photosynthesizing zinc sulfide edifices as cradles of life on Earth. Biol Direct 4:27.

57. Buckel W, and Thauer RK (2013). Energy conservation via electron bifurcating ferredoxin reduction and proton/ $/ \mathrm{Na}^{+}$translocating ferredoxin oxidation. Biochim Biophys Acta 1827: 94-113.
58. Thauer RK, Kaster AK, Seedorf H, Buckel W, and Hedderich R (2008). Methanogenic archaea: ecologically relevant differences in energy conservation. Nat Rev Microbiol. 6:579-59.

59. Schuchmann K, and Müller V (2014). Autotrophy at the thermodynamic limit of life: a model for energy conservation in acetogenic bacteria. Nat Rev Microbiol. 12:809-821.

60. Liu Y, Beer LL, and Whitman WB. (2012). Methanogens: a window into ancient sulfur metabolism. Trends Microbiol. 20:251 - 258.

61. Lever MA (2012). Acetogenesis in the energy-starved deep biosphere-a paradox? Front Microbiol 2:284.

62. Sleep NH, Meibom A, Fridriksson T, Coleman RG, and Bird DK (2004) $\mathrm{H}_{2}$-rich fluids from serpentinization: geochemical and biotic implications. Proc Natl Acad Sci U S A 101:12818-12823.

63. Ferry JG, House CH (2006). The step-wise evolution of early life driven by energy conservation. Mol Biol Evol 23:1286-1292.

64. Eck RV, and Dayhoff MO (1966). Evolution of the structure of ferredoxin based on living relics of primitive amino acid sequences. Science 152:363-366.

65. Fuchs $G$ (2011). Alternative pathways of carbon dioxide fixation: insights into the early evolution of life? Annu Rev Microbiol 65:631658.

66. Basen M, and Müller V (2016). "Hot" acetogenesis. Extremophiles $1-12$. 